\title{
Building Trust in RWE Will Support Clearer Regulatory Uses
}

\author{
Therapeutic Innovation \\ \& Regulatory Science \\ 2019, Vol. 53(4) 417-419 \\ (C) DIA 2019 \\ Article reuse guidelines: \\ sagepub.com/journals-permissions \\ DOI: $10.1177 / 2168479019859948$ \\ tirs.sagepub.com
}

Passage of the $21^{\text {st }}$ Century Cures Act of 2016 (Cures Act) required FDA to establish a program to evaluate and enhance the use of RWE on product effectiveness in its regulatory decision-making. ${ }^{1}$ Since then, academic researchers and multi-stakeholder collaborations by the National Academies of Sciences $^{2}$, the International Society of Pharmacoeconomics and Outcomes Research (ISPOR) ${ }^{3,4}$, the Drug Information Association, Friends of Cancer Research, and my own group, the Duke-Robert J. Margolis, MD, Center for Health Policy, have held public forums and published recommendations and whitepapers to bolster use of real world data (RWD) and real world evidence (RWE) in regulatory decision-making. The consensus from these groups has focused in critical areas to help improve the credibility of and trust in RWE: data quality, research design and methods, and transparency.

The December 2018 Framework for FDA's RWE Program ${ }^{5}$ established that these are among Agency's highest priority areas in determining whether RWD and the resultant RWE is fit-for-use to support regulatory decisions on effectiveness. While RWD use could improve the efficiency of traditional clinical trials (e.g., trial recruitment and stratification), the RWE program's scope is to evaluate how RWD used to generate RWE on product effectiveness can support approval of new indications for drugs or to help satisfy post-approval study requirements. ${ }^{6}$ FDA has some experience in using RWE to support regulatory decisions in cancers and rare diseases (primarily external controls in single-arm studies) as well as in post-market safety assessments (e.g., using the Sentinel System). ${ }^{7}$ However, the regulatory decisions contemplated in Cures and the focus of FDA's RWE Program are specifically to support changes to labeling about drug product effectiveness, including adding or modifying an indication, adding a new population, or adding comparative effectiveness or safety

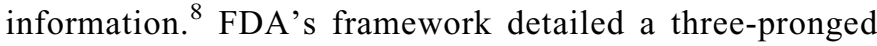
approach to evaluate RWE use including: "1) whether RWD are fit-for-use; 2) whether the trial or study design used to generate RWE can provide adequate scientific evidence to answer or help answer the regulatory question; and 3) whether the study conduct meets FDA regulatory requirements" as well as outlined supportive programmatic efforts.

Considering broader uses of RWE in regulatory decisions and subsequently reflecting that evidence in drug product labeling will enable more patient- and provider-relevant information to be available and communicated. This can include evidence of product effectiveness in patients reflective of real world clinical practice as well as on longer-term and more patient- relevant endpoints. This could also enable more cost-effective and efficient evidence generation. However, for RWE to be more fully embraced as a viable source of evidence in this context, much work is needed to come to consensus on how to ensure trust and credibility in the RWD and resultant RWE.

Improving Trust Through Data Quality: The FDA framework highlights the need to assess RWD fitness-for-use, including measuring data reliability (a function of data quality and data accrual) and data relevancy. FDA's experience in evaluating clinical trial data quality (and resulting comfort) is high. In contrast to randomized clinical trial (RCT) data, RWD sources, including claims, EHR data, secondary uses of registries, and patient-generated health data (e.g., from sensors or technologies that capture patient-reported outcomes) have significant heterogeneity within and between them. Subsequently, evaluating RWD quality by FDA reviewers will be challenging unless assessing and communicating fitness-for-use are standardized. Underpinning this challenge is that using RWD in this context is a secondary use of the data, and often requires complicated and non-standardized approaches for cleaning, transforming, and linking the data at various stages ${ }^{9}$ to make them "research-ready." To the end user of the evidence, the data curation process and subsequent bias introduction, as well as data loss and context, are often opaque. While the Agency has growing experience with some RWD types, it does not have clear standards or identified best practices for appropriate data quality assessment. The regulatory and research communities will to need to come to consensus, and build upon previous work done by groups like the Patient Centered Outcomes Research Institute and the Sentinel System, and develop tools for efficient and adequate assessment of data quality and ultimately fitness-for-use.

Improving Trust Through Research Design: FDA committed to developing guidance on considerations for designing randomized RWE studies (i.e., including pragmatic design elements), single arm trials with external control groups, and observational (non-interventional) studies that generate evidence of effectiveness for regulatory decisions. ${ }^{10}$ A public workshop in Washington, DC, on July 11 and 12 will discuss considerations for randomized designs ${ }^{11}$, including recruitment and enrollment approaches, facilitating intervention strategies, blinding issues, and outcomes assessment. While questions exist on how all three study designs can support regulatory decisions on effectiveness, perhaps the greatest uncertainty to making causal inferences are with observational designs, including prospective and retrospective database studies. 
Fortunately, FDA can draw on a wealth of research on improving credibility in observational designs. ${ }^{12}$ However, linking how these designs could lead to studies that are considered "adequate and well-controlled," and meet the statutory "substantial evidence" bar is still necessary. ${ }^{13,14}$ Given that the RWE Program is focused on using RWE for new regulatory decisions about drug products already on the market, how one or more new RWE studies are taken into account within a product's totality of the evidence (e.g., previous RCTs used for the original approval) must also be explored.

Improving Trust Through Transparency: Overall transparency of the data, study methods, and results, is critical for increasing RWE trust. However, for retrospective studies, the timing of the transparency is also critical to trust, in that study design and analysis must be pre-specified prior to study execution. ${ }^{15}$ It is conceivable that because the data exists prior the retrospective study initiation, a researcher could conduct analyses with different design parameters until a favorable result is found, and then develop a protocol to replicate and ultimately submit that finding. There are options for mitigating this concern of "cherry-picking," including replication of results in multiple different databases, conducting sensitivity analyses, and publicly registering the study protocol and analysis ahead of conducting the study. Building on past work ${ }^{16,17}$, ISPOR, in partnership the International Society for Pharmacoepidemiology, the National Pharmaceutical Council, and Duke-Margolis, have formed a multi-stakeholder "RWE Transparency Initiative Partnership" which is developing recommendations for how publically accessible pre-registration for retrospective RWE effectiveness studies, similar to ClinicalTrials.gov ${ }^{18}$, might help address these concerns through increasing transparency and ultimately research quality.

The highlighted steps will help improve RWE trust. However, to fully understand whether and how RWE can support regulatory decisions on effectiveness, we must recognize that this is a journey and that the broader regulatory and scientific communities, along with FDA, will need to learn along the way. In May, FDA released a draft guidance entitled Submitting Documents Using Real-World Data and Real-World Evidence to FDA for Drugs and Biologics. ${ }^{19}$ The guidance is intended to encourage sponsors to provide specific information about RWD and RWE when they are included in their submission to FDA, and will be used only for internal tracking purposes. Ideally, this will enable the Agency to examine and reflect on the circumstances in which the RWE were deemed sufficient to support the review process, regardless of the decision outcome. Communicating these learnings to the broader community would help in better designing RWE studies for regulatory submissions.

I hope you join us in November in Cambridge, Massachusetts for the Annual DIA Real World Evidence Conference to delve into these topics further.

I would like to thank my colleague Nirosha Mahendraratnam Lederer for her help in developing this editorial.

\author{
Gregory W. Daniel, PhD, MPH, RPh \\ Editor-in-Chief, \\ Therapeutic Innovation \& Regulatory Science
}

\section{References}

1. Public Law 114-225. Available at: https://www.congress.gov/ bill/114th-congress/house-bill/34/

2. National Academics of Sciences, Engineering, and Medicine. "Examining the Impact of Real-World Evidence on Medical Product Development: Proceedings of a Workshop Series." 6 Feb 2019. Available at: http://nationalacademies.org/hmd/reports/ 2019/examining-impact-real-world-evidence-on-medical-prod uct-development-proceedings.aspx?utm_source $=$ HMD + Email +List\&utm_campaign $=88$ b074a0b0-EMAIL_CAMPAIGN_ 2018_05_29_03_33_COPY_02\&utm_medium=email\&utm_ term $=0 \_211686812 \mathrm{e}-88 \mathrm{~b} 074 \mathrm{a} 0 \mathrm{~b} 0-180620349 \& \mathrm{mc} \_\mathrm{cid}=88 \mathrm{~b}$ 074a0b0\&mc_eid $=834 \mathrm{c} 54 \mathrm{a} 79 \mathrm{a}$

3. Berger, M.J, Sox, H., Willke, R.J., Brixner, D.L., Hans-Georg, E., Goettsch, W., Madigan, D., Makady, A., Schneeweiss, S., Tarricone, R., Wang, S.V., Watkins, J., and Mullins, C.D. (2017). Good Practices for Real-World Data Studies of Treatment and/ or Comparative Effectiveness: Recommendations from the Joint ISPOR-ISPE Special Task Force on Real-World Evidence in Health Care Decision Making, Pharmacoepidemiology and Drug Safety, 26(9):1033- 1039. doi:10.1002/pds.4297

4. Wang, S.V., Schneeweiss, S., Berger, M.L., Brown, J., de Vries, F., Douglas, I., Gagne, J.J., Gini, R., Klungel, O., Mullins, C.D., Nguyen, M.D., Rassen, J.A., Smeeth, L., and Sturkenboom, M. (2017). Reporting to Improve Reproducibility and Facilitate Validity Assessment in Healthcare Database Studies V1.0, Pharmacoepidemiology and Drug Safety, 26(9):1018-1032. doi:10. 1002/pds.4295.

5. Public Law 114-255. Available at: https://www.congress.gov/ bill/114th-congress/house-bill/34/

6. U.S. Food and Drug Administration. "Framework for FDA's Real-World Evidence Program.” December 2018. Available at: https://www.fda.gov/media/120060/download

7. U.S. Food and Drug Administration. "FDA's Sentinel Initiative." 21 May 2018. Available at: www.fda.gov/safety/fdas-sentinelinitiative.

8. U.S. Food and Drug Administration. "Framework for FDA's Real-World Evidence Program.” December 2018. Available at: https://www.fda.gov/media/120060/download

9. Duke-Margolis Center for Health Policy. Characterizing RWD Quality and Relevancy for Regulatory Purposes. 1 October 2018. Available at: https://healthpolicy.duke.edu/sites/default/ files/atoms/files/characterizing_rwd.pdf

10. U.S. Food and Drug Administration. "Framework for FDA's Real-World Evidence Program.” December 2018. Available at: https://www.fda.gov/media/120060/download

11. Leveraging Randomized Clinical Trials to Generate Real-World Evidence for Regulatory Purposes. Public Meeting. 11-12 July 2019. Available at: https://healthpolicy.duke.edu.events/lever 
aging-randomized-clinical-trials-generate-real-world-evdienceregulatory-purposes

12. Frank JM and Schneeweiss S. "When and How Can Real World Data Analyses Substitute for Randomized Controlled Trials?" Clin Pharmacol Ther. 2017 Dec;102(6):924-933.

13. 21 CFR 314.126

14. Section 505 of Federal Food, Drug, and Cosmetic Act.

15. U.S. Food and Drug Administration. "Framework for FDA's Real-World Evidence Program.” December 2018. Available at: https://www.fda.gov/media/120060/download

16. Berger, M.J, Sox, H., Willke, R.J., Brixner, D.L., Hans-Georg, E., Goettsch, W., Madigan, D., Makady, A., Schneeweiss, S., Tarricone, R., Wang, S.V., Watkins, J., and Mullins, C.D. (2017). Good Practices for Real-World Data Studies of Treatment and/ or Comparative Effectiveness: Recommendations from the Joint ISPOR-ISPE Special Task Force on Real-World Evidence in
Health Care Decision Making, Pharmacoepidemiology and Drug Safety, 26(9):1033- 1039. doi:10.1002/pds.4297.

17. Wang, S.V., Schneeweiss, S., Berger, M.L., Brown, J., de Vries, F., Douglas, I., Gagne, J.J., Gini, R., Klungel, O., Mullins, C.D., Nguyen, M.D., Rassen, J.A., Smeeth, L., and Sturkenboom, M. (2017). Reporting to Improve Reproducibility and Facilitate Validity Assessment in Healthcare Database Studies V1.0, Pharmacoepidemiology and Drug Safety, 26(9):1018-1032. doi:10.1002/pds.4295.

18. US National Library of Medicine. "ClinicalTrials.gov." Available at: https://clinicaltrials.gov/.

19. U.S. Food and Drug Administration. "Submitting Documents Utilizing Real-World Data and Real-World Evidence to FDA for Drugs and Biologics." Draft Guidance. 2019 May. Available at: https://www.fda.gov/regulatory-information/search-fda-gui dance-documents/submitting-documents-utilizing-real-worlddata-and-real-world-evidence-fda-drugs-and-biologics 